\title{
Mitrofanoff continent catheterizable conduits: top down or bottom up?
}

\author{
Berkowitz J, North AC, Tripp R, Gearhart JP, Lakshmanan Y
}

Division of Pediatric Urology, Brady Urological Institute, Johns Hopkins Medical Institutions, Baltimore, MD, USA

J Pediatr Urol. 2009; 5: 122-5

Objective: During augmentation and Mitrofanoff procedures, conduits are usually implanted into the posterior bladder wall. Anatomical considerations may necessitate an anterior conduit. To compare the relative drainage efficiency in patients with posterior and anterior conduits, we studied their rates of bladder stone formation and urinary tract infection (UTI).

Materials and Methods: A retrospective chart review identified exstrophy patients who underwent augmentation and Mitrofanoff between 1991 and 2003. Patients with 3 years or greater follow-up were included. Fifty-four patients fit this criterion, with a conduit implanted anteriorly (33) or posteriorly (21). We compared rates of bladder stone formation and UTI. Stomal revisions and the status of the bladder neck were also noted.

Results: Stone formation and UTI rates were higher in the anterior conduits, although only UTI showed a statistically significant difference. Patient demographics were similar between the two groups, including age and sex. The rates of stomal complications and the bladder neck status were also similar.

Conclusions: Patients with anterior conduits had an increased risk of UTI and bladder stone formation compared to those with posterior conduits, although this was not significant in the case of bladder stone rate. This may indicate sub-optimal bladder drainage and should be addressed with careful preoperative counseling and close follow-up.

\section{Editorial Comment}

A 12-year period from 1991-2003 was chosen and Mitrofanoff conduits were reviewed. All patients had greater than 3 years of continuous follow up. Fifty-four patients fit the criterion and 33 had the conduit anastomose to the augmented bladder anteriorly and 21 had their Mitrofanoff conduit placed posteriorly. Patients with cloacal exstrophy and spina bifida were excluded from this data base because it was felt that they had a higher risk of stone formation, which was one of the study criteria. Stomas were placed at the umbilicus. Patients were told to irrigate their augmented bladders daily with saline and had renal and bladder ultrasounds every three months for the first year and every six months thereafter.

Results showed that urinary tract infections were $36.3 \%$ in the anterior placed stomas and $9.5 \%$ in the posterior group and that was statistically significant. Bladder stones were also higher in the anterior placed conduit group at $48.4 \%$, but this did not reach statistical significance. Stomal complications were not statistically different and most of these were at the skin level. Stomal complications were common happening in over $42 \%$ of the patients, with an average of over 3 years of waiting for the stomal complications. The authors felt that the bladder was drained more poorly with the anterior placement of the conduits increasing the infection risk and the risk of stone disease. Many times placement of the conduit was dictated by the disease of the patient and the type of bowel utilized and prior scar tissue.

Augmentations in conduit placements are still fraught with complications. They solve many problems and bring on new problems. This is quite a high stone complication rate in these patients and stomal complications, although they are becoming more commonly noted. It seems that placing the catheterizable stoma in the most dependent portion in the augmented bladder has the best advantage and should be considered by urologic surgeons.

Dr. Brent W. Snow

Division of Urology

University of Utah Health Sci Ctr

Salt Lake City, Utah, USA

E-mail:brent.snow@hsc.utah.edu 